\title{
Atenção ao pré-natal: principais estratégias utilizadas durante a pandemia do COVID-19
}

Attention to the prenatal care: main strategies used during the COVID-19 pandemic

Atención prenatal: principales estrategias utilizadas durante la pandemia COVID-19

Giulia Carolina de Souza Santana ${ }^{1 *}$, Maria Clara Mota Souza do Amor¹, Bárbara Angélica Gómez Pérez¹.

\section{RESUMO}

Objetivo: Identificar as principais estratégias utilizadas na assistência ao pré-natal durante a pandemia do COVID-19 presentes na literatura. Métodos: Trata-se de uma revisão integrativa realizada através das bases de dados Literatura Latino-Americana e do Caribe em Ciências da Saúde (LILACS) e Medical Literature Analysis and Retrieval System Online (MEDLINE) a partir dos Descritores em Ciências Saúde: "COVID-19", "Pré-Natal" e "Gestação" e os Medical Subject Headings: "COVID-19", "Prenatal Care" e "Pregnancy", selecionados por intermédio do operador booleano AND. Dos 210 estudos disponibilizados, 15 foram selecionados, conforme os critérios de inclusão e exclusão pré-estabelecidos. Resultados: As principais estratégias utilizadas na assistência ao pré-natal durante o COVID-19, foram: utilização da telemedicina, continuação das consultas presenciais, porém evitando aglomerações e com todas as medidas de prevenção de contágio, adiamento da consulta em caso de a gestante apresentar algum sintoma e a qualificação dos profissionais de saúde. Considerações finais: A partir deste estudo foi possível identificar quais foram as principais estratégias utilizadas na assistência ao pré-natal durante a pandemia presentes na literatura. Porém, são necessários mais estudos sobre a temática, visto que ainda são poucos. Proporcionando uma assistência mais segura e respaldada na literatura científica.

Palavras-chave: COVID-19, Pré-natal, Gestação.

\begin{abstract}
Objective: Identify the main strategies used in the prenatal care during the COVID-19 pandemic present in the literature. Methods: It is a integrative review realized through database from Latin American and Caribbean literature in health sciences (LILACS) and Medical Subject Headings: "COVID-19", "Prenatal Care" and "Pregnancy", selected by intermediate of the boolean operator AND. Of the 210 studies available, 15 were selected, according to the inclusion and exclusion criteria pre-established. Results: The main strategies used in the prenatal care during the COVID-19, were: telemedicine, presential consults continuation, although avoiding agglomerations and with all preventive measures contagious, consultation postponement in case of the pregnant presentes symptoms and the qualification of health professionals. Final considerations: Through this study it was possible to identify what were the main strategies used in the prenatal care during the pandemic present in the literature. However, were needed more studies about the thematic, since there are still few. Providing an assistance safer and supported in scientific literature.
\end{abstract}

Key words: COVID-19, Prenatal care, Pregnancy.

\section{RESUMÉN}

Objetivo: Identificar las principales estrategias utilizadas en la atención prenatal durante la pandemia COVID19 presentes en la literatura. Métodos: Revisión integrativa realizada utilizando las bases de datos de Literatura Latinoamericana y del Caribe en Ciencias de la Salud (LILACS) y Sistema de Recuperación y Análisis de Literatura Médica en Línea (MEDLINE) de los Descriptores de Ciencias de la Salud: "COVID -19", "Prenatal". y "Embarazo" y los Medical Subject Headings: "COVID-19", "Prenatal Care" y "Pregnancy", seleccionados mediante el operador booleano AND. De los 210 estudios disponibles, 15 se seleccionaron según los criterios de inclusión y exclusión preestablecidos. Resultados: Las principales estrategias utilizadas

\footnotetext{
${ }^{1}$ Escola Bahiana de Medicina e Saúde Pública (EBMSP), Salvador - BA.
}

*E-mail: giuliasantana97@gmail.com 
en la atención prenatal durante el COVID-19 fueron: uso de telemedicina, continuación de las consultas presenciales, pero evitando el aglomeración y con todas las medidas para prevenir el contagio, postergación de la consulta en caso de que la gestante se presente cualquier síntoma y la calificación de los profesionales de la salud. Consideraciones finales: A partir de este estudio fue posible identificar las principales estrategias utilizadas en la atención prenatal durante la pandemia presentes en la literatura. Sin embargo, se necesitan más estudios sobre el tema, pues todavía son pocos. Ofreciendo asistencia más segura y respaldada por literatura científica.

Palabras clave: COVID-19, Atención prenatal, Embarazo.

\section{INTRODUÇÃO}

A gestação é um período sensível e principalmente desafiador na vida de uma mulher, desde o momento do planejamento, concepção e ao longo das fases do bebê. Nesse período, a mulher passa por uma série de alterações físicas, hormonais, sociais e psicológicas. Os níveis hormonais são elevados, entre eles o estrogênio, que ocasiona transtornos emocionais e principalmente o aumento da sensibilidade, fazendo com que a mulher fique mais vulnerável, necessitando de maior atenção e supervisão qualificada (MINISTÉRIO DA SAÚDE, 2012).

Durante o período gestacional a mulher precisa ser acompanhada de forma integral e humanizada e o prénatal se bem realizado atende a este objetivo, com o intuito de levantar todos os diagnósticos prévios, identificação de riscos, prevenção de agravos e ações de educação em saúde, podendo atuar nos pontos específicos para possibilitar a promoção da saúde ao bebê e a gestante, sendo preconizado pelo Ministério da saúde que esses aspectos sejam avaliados no pré-natal durante todas as fases gestacionais (MINISTÉRIO DA SAÚDE, 2013).

Nesse sentido, a atenção multiprofissional durante o pré-natal é de extrema importância para a qualidade do cuidado prestado à gestante, possibilitando diferentes olhares sobre essas práticas e atendendo a mesma de forma integral, interdisciplinar e acolhedora. Isto posto, o acolhimento é um ponto crucial no pré-natal, baseando-se no trabalho em equipe, permitindo o vínculo entre profissional e paciente e entre profissionais (TOMAZETTI BM, et al., 2018).

Entretanto, com o surgimento do novo coronavírus, houve mudanças significativas na assistência ao prénatal. Em virtude do isolamento, do medo, da insegurança e da ansiedade muitas gestantes que realizavam as consultas regularmente, deixaram de ter um acompanhamento contínuo e correto nesta fase, assim podendo prejudicar a sua saúde e consequentemente do seu feto (ARAÚJO D, et al., 2020).

Além disso, observou-se que gestantes e puérperas até a $14^{\text {a }}$ semana apresentam um maior risco de complicações nos quadros da doença, sendo incluídas como grupo de risco para a COVID-19. Por esse motivo, as gestantes precisam de maior atenção, devendo ser reforçados o distanciamento social, o uso de máscara e a higiene das mãos, assim como elaboração de novas estratégias que protejam esse grupo e que promovam a prevenção de riscos e a promoção da saúde durante o período da pandemia (MINISTÉRIO DA SAÚDE, 2020).

Nesse contexto, durante a pandemia, com a propagação do vírus, o medo do desconhecido, a falta de informações acerca das consequências para a gestação, além das preocupações com o momento do parto, a presença ou não de acompanhante, riscos durante a amamentação e risco de contaminação do bebê, são fatores que acarretam um sofrimento psíquico para essas mulheres durante esse momento tão delicado e importante (LÉLIS BDB, et al., 2020).

Diante do exposto, o presente estudo justifica-se pela necessidade de identificar dados atuais e presentes na literatura científica sobre as novas estratégias utilizadas na atenção pré-natal durante a pandemia do COVID-19, visto que muito se fala sobre a patologia, mas em associação a assistência pré-natal existem poucas publicações relativas às evidências científicas. Com isso, o objetivo deste artigo foi identificar as principais estratégias utilizadas na assistência ao pré-natal durante a pandemia do COVID-19 presentes na literatura. 


\section{MÉTODOS}

Trata-se de uma revisão integrativa da literatura, caracterizada por Souza MT, et al. (2010) como uma maneira de proporcionar a condensação do conhecimento e a integração da aplicação de resultados de estudos na prática. Segundo Botelho LLR, et al. (2011), a revisão integrativa é dividida em seis etapas, sendo elas: Identificação do tema e seleção da questão de pesquisa; Estabelecimento dos critérios de inclusão e exclusão; Identificação dos estudos pré-selecionados e selecionados; Categorização dos estudos selecionados; Análise e interpretação dos resultados e por fim apresentação da revisão/síntese do conhecimento.

O estudo sustentou-se a partir da seguinte questão norteadora: "Quais são as principais estratégias que estão sendo utilizadas na atenção ao pré-natal durante a pandemia do COVID-19 presentes na literatura, para assegurar a continuidade de um atendimento seguro e eficaz?". O levantamento dos artigos foi realizado entre os meses de agosto de 2020 a julho de 2021, através das bases de dados Literatura Latino-Americana e do Caribe em Ciências da Saúde (LILACS) e Medical Literature Analysis and Retrieval System Online (MEDLINE) a partir dos Descritores em Ciências Saúde (DeCS): "COVID-19", "Pré-Natal" e "Gestação" e os Medical Subject Headings (MeSH): "COVID-19", "Prenatal Care" e "Pregnancy", selecionados por intermédio do operador booleano $A N D$.

Foram considerados os seguintes critérios de inclusão: artigos originais, que contenham informações relativas ao tema estudado, publicados a partir de 2019, nos idiomas portugueses ou inglês, com texto completo disponível. E, como critérios de exclusão, artigos repetidos nas bases de dados, sendo mantidos em apenas uma base, incompletos, revisões de literatura, teses, dissertações, capítulos de livro e estudos que não abordavam a temática selecionada.

A partir dessa busca, foram encontrados 210 estudos. Após leitura dos títulos e resumos foi realizada a análise para identificar os estudos que se adequaram aos critérios de inclusão e exclusão pré-estabelecidos, 5 foram excluídos por não estarem disponíveis na íntegra, 125 foram excluídos por não abordarem a temática do estudo, 52 foram excluídos por não serem estudos primários e 1 foi excluído por não estar entre os idiomas selecionados. Sendo selecionados 27 estudos para leitura na íntegra. Após a leitura exaustiva, 12 artigos foram excluídos pela insuficiência de dados correspondente a resposta do objetivo, assim 15 artigos foram selecionados para compor a revisão (Figura 1).

Figura 1 - Fluxograma de resultados das buscas nas bases de dados.
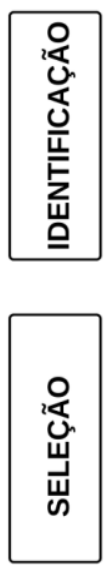

Excluídos por não estarem disponíveis na integra $(n=5)$
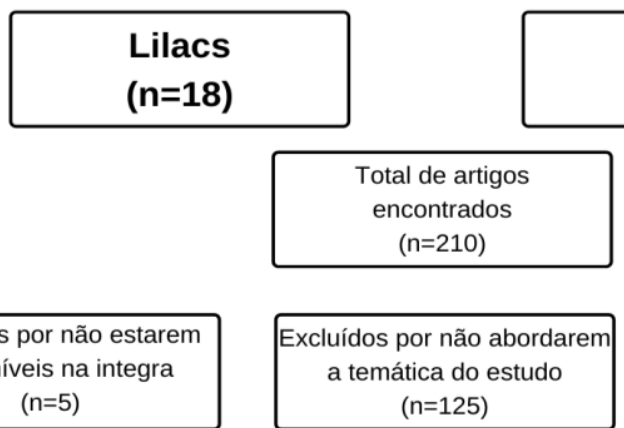
$(n=125)$

Excluídos por estarem fora dos idiomas selecionados $(n=1)$

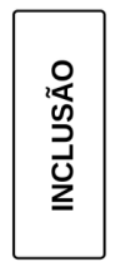

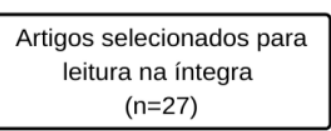

$(n=27)$

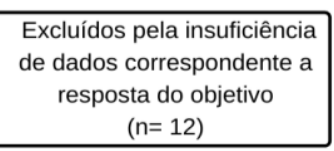
$(n=12)$

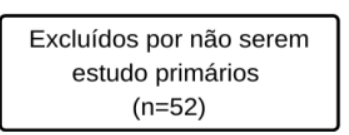

Medline

$(n=192)$

Fonte: Santana GCDS, et al., 2021. 
A análise de dados foi realizada com o auxílio do banco de dados elaborado a partir do programa Excel versão 2016, onde foram analisados e apresentados em forma de resultados, demonstrando assim os objetivos da pesquisa. Esses resultados foram desenvolvidos tendo em vista todo o embasamento científico utilizado durante o processo de construção.

O presente estudo não precisou ser submetido ao Comitê de Ética em Pesquisa (CEP) por se tratar de uma revisão de literatura, tendo como referências bases de dados públicos, respeitando, porém os preceitos éticos estabelecidos na resolução número 466/2012 do Conselho Nacional de Saúde (BRASIL, 2012).

\section{RESULTADOS E DISCUSSÃO}

Logo após a seleção dos artigos incluídos na amostra final, os mesmos foram organizados e caracterizados quanto ao título, autores, ano de publicação, objetivo, tipo de pesquisa, local do estudo e principais resultados (Quadro 1).

De acordo com o Quadro 1, foram encontrados artigos que respondiam ao objetivo proposto publicados nos anos de 2020 e 2021, mas o ano com maior número de publicações foi o de 2021 totalizando 9 artigos dos 15 selecionados, em virtude de ser um assunto muito recente. Além disso, o COVID-19 teve seu surgimento no final do ano de 2019 , e estudos sobre a temática são atuais, fato importante para atualização do assunto em questão.

O tipo de estudo predominante foi o estudo transversal, sendo que dos 15 artigos selecionados, 5 destes utilizaram essa metodologia, o que pode ser justificado pelo fato desse tipo de estudo ser empregue para examinar dados representativos da população em um momento específico, em que condições e impactos são assistidos em um tempo estabelecido, logo, esse é precisamente o objetivo da pesquisa, analisar a assistência ao pré-natal para com a gestantes durante a pandemia do COVID-19, assim descrevendo as estratégias utilizadas neste período.

As outras 10 publicações se dividiram em estudo ecológico longitudinal observacional ( 1 artigo), ensaio clínico randomizado ( 1 artigo), estudo de coorte (1 artigo), estudo retrospectivo (1 artigo), estudo observacional (2 artigos), estudo ecológico retrospectivo (1 artigo), qualitativo (2 artigos) e pesquisa exploratória de análise documental (1 artigo).

Estudos de diversos países foram analisados, como os Estados Unidos, que foi o mais predominante na realização das pesquisas com 7 publicações, mas também foram observados estudos de países como: Reino Unido (1 artigo), Turquia (2 artigos), Brasil (2 artigos), China (1 artigo), Japão (1 artigos), e Espanha (1 artigo).

Logo após a seleção dos artigos incluídos na amostra final, os mesmos foram organizados e caracterizados quanto ao título e considerações finais (Quadro 2). 
Quadro 1 - Caracterização dos artigos quanto ao título, autores, ano de publicação, objetivo, tipo de pesquisa, local do estudo e principais resultados.

\begin{tabular}{|c|c|c|c|c|c|}
\hline $\mathbf{N}^{\circ}$ & Título & Autores/ Ano & Objetivo & $\begin{array}{l}\text { Tipo de estudo/ Local do } \\
\text { estudo }\end{array}$ & Principais resultados \\
\hline $\mathrm{A} 1$ & $\begin{array}{l}\text { Um novo modelo multiplataforma para } \\
\text { atendimento ambulatorial pré-natal e } \\
\text { pós-parto em uma coorte de pacientes } \\
\text { obstétricas afetadas por COVID-19. }\end{array}$ & $\begin{array}{l}\text { Gutiérrez MM, et al. } \\
\text { (2021) }\end{array}$ & $\begin{array}{l}\text { Descrever um novo modelo de atendimento } \\
\text { multiplataforma que foi desenvolvido } \\
\text { durante o surgimento da pandemia para } \\
\text { atender às necessidades de pacientes } \\
\text { obstétricas que sofrem de COVID-19 } \\
\text { durante a gravidez, trabalho de parto ou } \\
\text { pós-parto. }\end{array}$ & $\begin{array}{l}\text { Estudo ecológico } \\
\text { longitudinal observacional. } \\
\text { Espanha. }\end{array}$ & $\begin{array}{l}\text { Foi evidenciado que as estratégias } \\
\text { mencionadas no artigo foram: agendamento de } \\
\text { consultas semanais e ligações telefônicas, } \\
\text { juntamente com alguns serviços presenciais } \\
\text { em sua maioria, vários procedimentos na } \\
\text { mesma visita (exame clínico, ultrassom e } \\
\text { exames de sangue). }\end{array}$ \\
\hline A2 & $\begin{array}{l}\text { Efeitos da atenção plena em pacientes } \\
\text { obstétricos e ginecológicos durante a } \\
\text { pandemia da doença coronavírus de } \\
2019 \text { (COVID-19): um ensaio clínico } \\
\text { randomizado }\end{array}$ & $\begin{array}{l}\text { Smith RB, et al. } \\
(2021)\end{array}$ & $\begin{array}{l}\text { Avaliar o efeito de um aplicativo de } \\
\text { meditação móvel baseado no consumidor } \\
\text { (app) no bem-estar de pacientes } \\
\text { ambulatoriais de obstetrícia e ginecologia } \\
\text { durante a pandemia de coronavírus } 2019 \text {. }\end{array}$ & $\begin{array}{l}\text { Ensaio clínico } \\
\text { randomizado. } \\
\text { Estados Unidos. }\end{array}$ & $\begin{array}{l}\text { Um aplicativo foi criado como uma das } \\
\text { estratégias para o cuidado com as gestantes } \\
\text { para redução de estresse, depressão, } \\
\text { ansiedade e distúrbios do sono. }\end{array}$ \\
\hline A3 & $\begin{array}{l}\text { Comparação entre visitas pré-natais } \\
\text { virtuais presenciais e apenas com áudio } \\
\text { e resultados perinatais. }\end{array}$ & $\begin{array}{l}\text { Duryea EL, et al. } \\
\qquad(2021)\end{array}$ & $\begin{array}{l}\text { Explorar a associação do pré-natal virtual } \\
\text { apenas com áudio com os resultados } \\
\text { perinatais. }\end{array}$ & $\begin{array}{l}\text { Estudo de coorte. Estados } \\
\text { Unidos. }\end{array}$ & $\begin{array}{l}\text { O pré-natal virtual com áudio foi uma estratégia } \\
\text { utilizada para o cuidado com as gestantes, } \\
\text { trazendo resultados positivos significativos. } \\
\text { Mulheres que deram à luz em } 2020 \text { após a } \\
\text { implementação de visitas virtuais pré-natais } \\
\text { apenas com audio não tiveram resultados } \\
\text { perinatais mais adversos em comparação com } \\
\text { as mulheres que deram à luz em } 2019 \text {. }\end{array}$ \\
\hline A4 & $\begin{array}{l}\text { Efeito do processo pandêmico COVID- } \\
19 \text { em procedimentos de diagnóstico } \\
\text { pré-natal. }\end{array}$ & Ozalp M, et al. (2020) & $\begin{array}{l}\text { Avaliar a acessibilidade de mulheres } \\
\text { grávidas à triagem pré-natal e testes } \\
\text { diagnósticos durante o processo } \\
\text { pandêmico CovID-19 e analisar o efeito do } \\
\text { processo pandêmico nas taxas de } \\
\text { aceitação-rejeição de procedimentos } \\
\text { diagnósticos fetais para gestações de alto } \\
\text { risco. }\end{array}$ & $\begin{array}{l}\text { Estudo transversal. } \\
\text { Turquia. }\end{array}$ & $\begin{array}{l}\text { Como resultado, há uma diminuição notável no } \\
\text { número de diagnósticos pré-natais e exames de } \\
\text { rastreamento durante a pandemia de COVID- } \\
19 \text {. No entanto, serviços completos de cuidados } \\
\text { pré-natais têm grande importância para a saúde } \\
\text { materna e fetal. } \\
\text { Assim, como estratégia utilizou-se a triagem } \\
\text { pré-natal e testes diagnósticos. }\end{array}$ \\
\hline A5 & $\begin{array}{l}\text { Viabilidade e segurança de telemedicina } \\
\text { materna iniciada com urgência em } \\
\text { resposta à disseminação de COVID-19: } \\
\text { um relatório de } 1 \text { mês. }\end{array}$ & $\begin{array}{l}\text { Nakagawa K, et al, } \\
(2020)\end{array}$ & $\begin{array}{l}\text { Investigar a segurança da telemedicina } \\
\text { materna iniciada com urgência na } \\
\text { prevenção da disseminação da infecção por } \\
\text { coronavírus. }\end{array}$ & $\begin{array}{l}\text { Estudo retrospectivo. } \\
\text { Japão. }\end{array}$ & $\begin{array}{l}\text { As intervenções de telemedicina foram } \\
\text { fornecidas e utilizadas como estratégias para o } \\
\text { atendimento do pré-natal, utilizando-se } \\
\text { consultas online. }\end{array}$ \\
\hline A6 & $\begin{array}{l}\text { A adoção da telessaúde no atendimento } \\
\text { pré-natal e nas atitudes do provedor } \\
\text { durante a pandemia do COVID-19 na } \\
\text { cidade de Nova York: um quantitativo e } \\
\text { Análise qualitativa. }\end{array}$ & $\begin{array}{l}\text { Madden N, et al. } \\
(2020)\end{array}$ & $\begin{array}{l}\text { Determinar em que grau o cuidado pré- } \\
\text { natal foi capaz de fazer a transição para } \\
\text { telessaúde em práticas pré-natais } \\
\text { associadas a dois hospitais afiliados em } \\
\text { Nova York durante a nova pandemia de } \\
\text { doença coronavírus } 2019 \text {. }\end{array}$ & $\begin{array}{l}\text { Estudo observacional. } \\
\text { Estados Unidos. }\end{array}$ & $\begin{array}{l}\text { Durante o período do estudo, a estratégia } \\
\text { escolhida para dar continuidade do cuidado foi } \\
\text { a telessaúde. O acesso à tecnologia e a } \\
\text { realizacãa de visitas, documentação e } \\
\text { acompanhamento usando o prontuário } \\
\text { eletrônico de telessaúde foram todos vistos } \\
\text { com bons olhos pelos provedores. }\end{array}$ \\
\hline
\end{tabular}




\begin{tabular}{|c|c|c|c|c|c|}
\hline $\mathbf{N}^{\circ}$ & Título & Autores/ Ano & Objetivo & $\begin{array}{c}\text { Tipo de estudo/ Local do } \\
\text { estudo }\end{array}$ & Principais resultados \\
\hline A7 & $\begin{array}{l}\text { Impacto da pandemia COVID-19 no pré- } \\
\text { natal, diabetes e consultas médicas no } \\
\text { Sistema Único de Saúde. }\end{array}$ & $\begin{array}{l}\text { Chisini LA, et al. } \\
\qquad(2021)\end{array}$ & $\begin{array}{l}\text { Descrever o impacto da pandemia COVID- } \\
19 \text { na Atenção Primária à Saúde no Brasil. }\end{array}$ & $\begin{array}{l}\text { Estudo ecológico } \\
\text { retrospectivo; Brasil. }\end{array}$ & $\begin{array}{l}\text { As soluções e estratégias apontadas pelos } \\
\text { autores permeiam a adoção de modelos de } \\
\text { rastreamento de pacientes, a utilização de } \\
\text { prontuários eletrônicos adequados, ampliação } \\
\text { do acesso à consulta médica por meio da } \\
\text { utilização de sistemas de telemedicina, } \\
\text { desenvolvimento de protocolos específicos } \\
\text { para o atendimento de doenças não } \\
\text { transmissíveis específicas para a duração da } \\
\text { pandemia. Fundamentalmente, deve-se ter em } \\
\text { mente os atributos essenciais da APS: acesso, } \\
\text { longitudinalidade, integralidade e coordenação } \\
\text { do cuidado. }\end{array}$ \\
\hline A8 & $\begin{array}{l}\text { Uso de aplicativos móveis por mulheres } \\
\text { grávidas e níveis de angústia da } \\
\text { gravidez durante a pandemia de } \\
\text { COVID-19. }\end{array}$ & $\begin{array}{l}\text { Sat SO e Sozbir SY } \\
\qquad(2021)\end{array}$ & $\begin{array}{l}\text { Identificar o uso de aplicativos móveis por } \\
\text { mulheres grávidas no recebimento de } \\
\text { informações de saúde, aconselhamento e } \\
\text { cuidados de saúde durante a pandemia de } \\
\text { COVID-19 e seus níveis de angústia } \\
\text { durante a gravidez. }\end{array}$ & $\begin{array}{l}\text { Estudo transversal } \\
\text { descritivo. } \\
\text { Turquia. }\end{array}$ & $\begin{array}{l}\text { Como estratégia a ser utilizada para o cuidado } \\
\text { pré-natal, foi utilizado por mulheres grávidas } \\
\text { aplicativos móveis para obter informações } \\
\text { sobre consultas e exames. }\end{array}$ \\
\hline A9 & $\begin{array}{l}\text { Experiência de mães grávidas usando } \\
\text { um serviço de suporte pré-natal } \\
\text { baseado em mídia social durante o } \\
\text { lockdown da COVID-19 no Reino Unido: } \\
\text { resultados de uma pesquisa com } \\
\text { usuários. }\end{array}$ & $\begin{array}{l}\text { Chatwin J, et al. } \\
\qquad(2021)\end{array}$ & $\begin{array}{l}\text { Explorar as experiências de mães que } \\
\text { estavam usando um serviço de suporte pré- } \\
\text { natal baseado em mídia social do National } \\
\text { Health Service (NHS), durante os estágios } \\
\text { iniciais do bloqueio COVID-19 no Reino } \\
\text { Unido. }\end{array}$ & $\begin{array}{l}\text { Estudo Qualitativo. Reino } \\
\text { Unido. }\end{array}$ & $\begin{array}{l}\text { A estratégia foi implementada a partir de um } \\
\text { aplicativo de informações sobre o pré-natal, } \\
\text { com o suporte do National Health Service } \\
\text { (NHS), nesse aplicativo as mulheres poderiam } \\
\text { tirar as dúvidas sobre o momento da gravidez, } \\
\text { parto, amamentação, além de terem contato } \\
\text { com outras grávidas e parteiras. O acesso } \\
\text { online foi visto como uma ajuda para preencher } \\
\text { uma lacuna durante o lockdown. }\end{array}$ \\
\hline A 10 & $\begin{array}{l}\text { Adesão e aceitabilidade de consultas de } \\
\text { telessaúde para pacientes obstétricas } \\
\text { de alto risco durante a pandemia do } \\
\text { coronavírus } 2019 \text {. }\end{array}$ & $\begin{array}{l}\text { Jeganathan S, et al. } \\
\qquad(2020)\end{array}$ & $\begin{array}{l}\text { Descrever as atitudes do paciente e do } \\
\text { provedor em relação à telessaúde para a } \\
\text { prestação de cuidados obstétricos de alto } \\
\text { risco em um grande sistema de saúde com } \\
\text { configurações urbanas e suburbanas. }\end{array}$ & $\begin{array}{l}\text { Estudo Transversal } \\
\text { Estados Unidos }\end{array}$ & $\begin{array}{l}\text { Foi realizada a implementação do serviço de } \\
\text { telessaúde para atender mulheres com } \\
\text { gravidez de alto risco, sendo assim após essa } \\
\text { estratégia ser utilizada o número de consultas } \\
\text { desmarcadas e o número de não } \\
\text { comparecimento reduziu no serviço. }\end{array}$ \\
\hline A 11 & $\begin{array}{l}\text { Perspectivas do paciente em visitas pré- } \\
\text { natais virtuais apenas com áudio em } \\
\text { meio à pandemia de síndrome } \\
\text { respiratória aguda grave do Coronavírus } \\
2 \text {. }\end{array}$ & $\begin{array}{l}\text { Holcomb D, et al. } \\
\qquad(2020)\end{array}$ & $\begin{array}{l}\text { Avaliar a satisfação do paciente após a } \\
\text { integração de visitas virtuais apenas de } \\
\text { áudio em um cronograma de atendimento } \\
\text { pré-natal pré-existente em um grande } \\
\text { sistema com base no condado durante a } \\
\text { pandemia de síndrome respiratória aguda } \\
\text { grave coronavírus } 2 \text { (SARS-CoV-2). }\end{array}$ & $\begin{array}{l}\text { Pesquisa transversal. } \\
\text { Estados Unidos. }\end{array}$ & $\begin{array}{l}\text { Consultas pré-natais virtuais apenas com áudio } \\
\text { foram incluídas no serviço a fim de evitar a } \\
\text { infecção pelo COVID-19. As consultas apenas } \\
\text { por áudio também tiveram uma maior } \\
\text { aceitabilidade. A maioria das pacientes preferiu } \\
\text { uma combinação de visitas presenciais e } \\
\text { virtuais para o cuidado pré-natal, e as pacientes } \\
\text { relataram muitos benefícios com as visitas } \\
\text { virtuais. }\end{array}$ \\
\hline
\end{tabular}




\begin{tabular}{|c|c|c|c|c|c|}
\hline $\mathbf{N}^{\circ}$ & Título & Autores/ Ano & Objetivo & $\begin{array}{c}\text { Tipo de estudo/ Local do } \\
\text { estudo }\end{array}$ & Principais resultados \\
\hline A 12 & $\begin{array}{l}\text { Telessaúde para gestações de alto risco } \\
\text { no cenário da pandemia COVID-19. }\end{array}$ & Aziz A, et al. (2020) & $\begin{array}{l}\text { Consolidar a triagem pré-natal, vigilância e } \\
\text { exames presenciais em menos visitas } \\
\text { presenciais, mantendo o acesso do } \\
\text { paciente a cuidados pré-natais contínuos e } \\
\text { consultas de subespecialidade por meio de } \\
\text { visitas virtuais de telessaúde. }\end{array}$ & $\begin{array}{l}\text { Estudo observacional. } \\
\text { Estados Unidos. }\end{array}$ & $\begin{array}{l}\text { A abordagem geral escolhida pelo serviço foi } \\
\text { aplicar a telessaúde no cuidado pré-natal em } \\
\text { gestantes de alto risco. Essa abordagem } \\
\text { envolveu a consolidação de testes e serviços } \\
\text { que precisavam ser realizados pessoalmente } \\
\text { durante a realização de aconselhamento } \\
\text { durante as sessões. A estrutura proposta para } \\
\text { o atendimento pré-natal de telessaúde elimina } \\
\text { aproximadamente metade das visitas pessoais } \\
\text { para pacientes de baixo risco. }\end{array}$ \\
\hline A 13 & $\begin{array}{l}\text { Estressores, enfrentamento e recursos } \\
\text { necessários durante a pandemia } \\
\text { COVID-19 em uma amostra de } \\
\text { mulheres perinatais. }\end{array}$ & $\begin{array}{l}\text { Barbosa-Leiker C, et } \\
\text { al. (2021) }\end{array}$ & $\begin{array}{l}\text { Avaliar estressores, comportamentos de } \\
\text { enfrentamento e recursos necessários em } \\
\text { relação à pandemia de COVID-19 em uma } \\
\text { amostra de } 162 \text { mulheres perinatais nos } \\
\text { Estados Unidos. }\end{array}$ & $\begin{array}{l}\text { Quantitativo e qualitativo. } \\
\text { Estados Unidos. }\end{array}$ & $\begin{array}{l}\text { Os participantes que relataram faltar às } \\
\text { consultas de pré-natal, fizeram o uso da } \\
\text { telemedicina para obter esses serviços. Sendo } \\
\text { a melhor estratégia encontrada pelo serviço. }\end{array}$ \\
\hline A 14 & $\begin{array}{l}\text { O surto do coronavírus na China: } \\
\text { percepções de risco, conhecimento e } \\
\text { fontes de informação entre mulheres } \\
\text { pré-natais e pós-natais. }\end{array}$ & Lee T, et al. (2021) & $\begin{array}{l}\text { Examinar as percepções de risco, } \\
\text { conhecimento e fontes de informação } \\
\text { relacionadas ao COVID-19 entre mulheres } \\
\text { chinesas pré e pós-natal durante a fase } \\
\text { inicial da pandemia do COVID-19. }\end{array}$ & $\begin{array}{l}\text { Estudo Transversal. } \\
\text { China. }\end{array}$ & $\begin{array}{l}\text { As três principais fontes de onde obtiveram } \\
\text { informações sobre o COVID-19 foram médicos, } \\
\text { enfermeiras / parteiras e a televisão, e eles } \\
\text { depositaram um alto nível de confiança nessas } \\
\text { fontes. Sendo assim, observa-se a importância } \\
\text { de ter como uma das estratégias o } \\
\text { aprimoramento e a qualificação dos } \\
\text { profissionais. }\end{array}$ \\
\hline A 15 & $\begin{array}{l}\text { Atenção perinatal na pandemia da } \\
\text { COVID-19: análise de diretrizes e } \\
\text { protocolos nacionais }\end{array}$ & $\begin{array}{l}\text { Stofel NS, et al. } \\
\qquad(2021)\end{array}$ & $\begin{array}{l}\text { Analisar à luz da experiência positiva na } \\
\text { perinatalidade, protocolos e diretrizes } \\
\text { brasileiras acerca da atenção à saúde } \\
\text { perinatal durante a pandemia da COVID-19 }\end{array}$ & $\begin{array}{l}\text { Pesquisa exploratória de } \\
\text { análise documental. Brasil. }\end{array}$ & $\begin{array}{l}\text { As diretrizes e protocolos trazem como } \\
\text { estratégias propostas dentro da Atenção ao } \\
\text { Pré-natal, a Atenção Primária à Saúde (APS) } \\
\text { como responsável por realizar a triagem das } \\
\text { gestantes, recomendando o adiamento dos } \\
\text { procedimentos eletivos por } 14 \text { dias em casos } \\
\text { suspeitos ou confirmados, orientando a mulher } \\
\text { a realizar isolamento domiciliar e observar a } \\
\text { evolução dos sintomas. }\end{array}$ \\
\hline
\end{tabular}


Quadro 2 - Caracterização dos artigos quanto ao título e conclusão.

\begin{tabular}{|c|c|c|}
\hline No $^{\circ}$ & Título & Considerações finais \\
\hline A1 & $\begin{array}{l}\text { Um novo modelo multiplataforma para atendimento ambulatorial pré-natal e } \\
\text { pós-parto em uma coorte de pacientes obstétricas afetadas por COVID-19 }\end{array}$ & $\begin{array}{l}\text { Os programas de telemedicina são uma ferramenta forte e reproduzível para chegar à população grávida } \\
\text { afetada pelo COVID-19, para avaliar seus sintomas e gravidade e para registrar os sintomas relacionados à } \\
\text { gravidez em regime ambulatorial e após a alta hospitalar. }\end{array}$ \\
\hline A2 & $\begin{array}{l}\text { Efeitos da atenção plena em pacientes obstétricos e ginecológicos durante } \\
\text { a pandemia da doença coronavírus de } 2019 \text { (COVID-19): um ensaio clínico } \\
\text { randomizado. }\end{array}$ & $\begin{array}{l}\text { Pacientes ambulatoriais de obstetrícia e ginecologia que usaram o aplicativo de meditação móvel baseado } \\
\text { no consumidor prescrito durante a pandemia de COVID-19 tiveram reduções significativas no estresse } \\
\text { percebido, depressão, ansiedade e distúrbios do sono em comparação com o tratamento padrão. }\end{array}$ \\
\hline A3 & $\begin{array}{l}\text { Comparação entre visitas pré-natais virtuais presenciais e apenas com } \\
\text { áudio e resultados perinatais. }\end{array}$ & $\begin{array}{l}\text { Visitas virtuais apenas de áudio proporcionam o cuidado necessário e completo em uma população sem } \\
\text { acesso imediato aos recursos e conhecimento tecnológico necessários para se engajar em visitas de vídeo. } \\
\text { Apesar disso, enfrentamos um dilema: as consultas pré-natais virtuais apenas com áudio são atualmente } \\
\text { consideradas aceitáveis como medidas de emergência, mas não como consultas completas para fins de } \\
\text { reembolso. Nossos resultados sugerem segurança, eficácia e legitimidade de uma plataforma de } \\
\text { atendimento pré-natal virtual síncrona, apenas com áudio, e defendem a equidade no reembolso em paralelo } \\
\text { às visitas pessoais. }\end{array}$ \\
\hline A4 & $\begin{array}{l}\text { Efeito do processo pandêmico COVID-19 em procedimentos de diagnóstico } \\
\text { pré-natal. }\end{array}$ & $\begin{array}{l}\text { Chama a atenção a diminuição do número de exames de diagnóstico e rastreamento pré-natal durante a } \\
\text { pandemia de COVID-19. Os serviços de cuidado pré-natal são um problema sério que não pode ser } \\
\text { superado por quaisquer deficiências nos cuidados maternos e fetais }\end{array}$ \\
\hline A5 & $\begin{array}{l}\text { Viabilidade e segurança de telemedicina materna iniciada com urgência em } \\
\text { resposta à disseminação de COVID-19: um relatório de } 1 \text { mês. }\end{array}$ & $\begin{array}{l}\text { A telemedicina materna pode ser conduzida com segurança em mulheres grávidas que correm o risco de } \\
\text { ter um distúrbio subjacente ou anomalia fetal } 1 \text { mês após o início da tentativa. Deve ser considerada como } \\
\text { uma forma de cuidado médico materno para prevenir a propagação da COVID-19. }\end{array}$ \\
\hline A6 & $\begin{array}{l}\text { A adoção da telessaúde no atendimento pré-natal e nas atitudes do } \\
\text { provedor durante a pandemia do COVID-19 na cidade de Nova York: um } \\
\text { quantitativo e Análise qualitativa. }\end{array}$ & $\begin{array}{l}\text { A telessaúde foi rapidamente implementada no cenário da pandemia COVID- } 19 \text { e foi vista com bons olhos } \\
\text { pelos provedores. Barreiras limitadas ao atendimento foram observadas para práticas que atendem } \\
\text { pacientes com seguro comercial. }\end{array}$ \\
\hline A7 & $\begin{array}{l}\text { Impacto da pandemia COVID-19 no pré-natal, diabetes e consultas médicas } \\
\text { no Sistema Único de Saúde. }\end{array}$ & $\begin{array}{l}\text { Os achados mostraram uma redução nos procedimentos de pré-natal, diabetes e consultas médicas } \\
\text { realizadas na Atenção Primária à Saúde no Brasil, após o início da pandemia do COVID-19. }\end{array}$ \\
\hline A8 & $\begin{array}{l}\text { Uso de aplicativos móveis por mulheres grávidas e níveis de angústia da } \\
\text { gravidez durante a pandemia de COVID-19 (Coronavírus). }\end{array}$ & $\begin{array}{l}\text { Este estudo ajudou a compreender o impacto da pandemia no sofrimento da gravidez e o uso de aplicativos } \\
\text { móveis de saúde por mulheres grávidas durante a pandemia. Além disso, nossos resultados indicam uma } \\
\text { diminuição no número de mulheres grávidas recebendo serviços de saúde durante este período. Os } \\
\text { aplicativos móveis de saúde parecem ser utilizáveis para acompanhamento pré-natal porque os aplicativos } \\
\text { móveis são comuns entre mulheres grávidas durante a pandemia de COVID-19. }\end{array}$ \\
\hline
\end{tabular}




\begin{tabular}{|c|c|c|}
\hline $\mathbf{N}^{\circ}$ & Título & Considerações finais \\
\hline A9 & $\begin{array}{l}\text { Experiência de mães grávidas usando um serviço de suporte pré-natal } \\
\text { baseado em mídia social durante o lockdown da COVID-19 no Reino Unido: } \\
\text { resultados de uma pesquisa com usuários }\end{array}$ & $\begin{array}{l}\text { O estudo sugere que as mães grávidas encontraram uma abordagem baseada nas redes sociais bem } \\
\text { posicionadas para fornecer cuidados pré-natais e apoio durante a pandemia COVID-19. }\end{array}$ \\
\hline A10 & $\begin{array}{l}\text { Adesão e aceitabilidade de consultas de telessaúde para pacientes } \\
\text { obstétricas de alto risco durante a pandemia do coronavírus } 2019 \text {. }\end{array}$ & $\begin{array}{l}\text { A implantação de um modelo de telessaúde na obstetrícia de alto risco tem o potencial de melhorar o acesso } \\
\text { à atenção obstétrica de alto risco, por meio da redução do índice de faltas às consultas. Tanto os pacientes } \\
\text { quanto os provedores pesquisados expressaram um alto índice de satisfação com as visitas de telessaúde } \\
\text { e o desejo de integrar a telessaúde ao modelo tradicional de atendimento obstétrico de alto risco. }\end{array}$ \\
\hline A11 & $\begin{array}{l}\text { Perspectivas do paciente em visitas pré-natais virtuais apenas com áudio } \\
\text { em meio à pandemia de síndrome respiratória aguda grave do Coronavírus } \\
2\end{array}$ & $\begin{array}{l}\text { Visitas pré-natais virtuais apenas com áudio - como um complemento às visitas pré-natais presenciais - têm } \\
\text { vantagens específicas e distintas em comparação com a telessaúde com vídeo em uma população } \\
\text { vulnerável de mulheres e oferecem uma opção viável para aumentar o acesso aos cuidados. }\end{array}$ \\
\hline A12 & $\begin{array}{l}\text { Telessaúde para gestações de alto risco no cenário da pandemia COVID- } \\
19\end{array}$ & $\begin{array}{l}\text { Em conclusão, embora as visitas virtuais de telessaúde não substituam totalmente os encontros pessoais } \\
\text { durante o cuidado pré-natal, elas oferecem um meio de reduzir a exposição do paciente e do provedor ao } \\
\text { COVID-19, ao mesmo tempo em que fornecem testes e serviços presenciais consolidados. Os regimes de } \\
\text { telessaúde para atendimento pré-natal podem ser modificados para pacientes de alto risco para garantir que } \\
\text { recebam acesso adequado a testes fetais e exames de ultrassom, consultores não obstétricos e seus } \\
\text { provedores de obstetrícia. }\end{array}$ \\
\hline A13 & $\begin{array}{l}\text { Estressores, enfrentamento e recursos necessários durante a pandemia } \\
\text { COVID-19 em uma amostra de mulheres perinatais }\end{array}$ & $\begin{array}{l}\text { Para melhor apoiar a saúde mental das mulheres perinatais durante a pandemia de COVID-19, os } \\
\text { profissionais de saúde devem se envolver em conversas sobre o acesso aos recursos necessários para } \\
\text { cuidar de recém-nascidos, encaminhar os pacientes para serviços de aconselhamento (que podem ser } \\
\text { fornecidos online / por telefone) e grupos de apoio virtual, e fazer uma triagem consistente de mulheres } \\
\text { grávidas quanto a fatores estressantes. }\end{array}$ \\
\hline A14 & $\begin{array}{l}\text { O surto do coronavírus na China: percepções de risco, conhecimento e } \\
\text { fontes de informação entre mulheres pré-natais e pós-natais. }\end{array}$ & $\begin{array}{l}\text { As presentes descobertas oferecem informações valiosas para profissionais de saúde, incluindo parteiras, } \\
\text { que atuam na linha de frente e prestam atendimento a mulheres grávidas. Embora os participantes tivessem } \\
\text { um conhecimento adequado sobre o COVID-19, eles interpretaram mal algumas das recomendações da } \\
\text { Organização Mundial da Saúde. }\end{array}$ \\
\hline A15 & $\begin{array}{l}\text { Atenção perinatal na pandemia da COVID-19: análise de diretrizes e } \\
\text { protocolos nacionais. }\end{array}$ & $\begin{array}{l}\text { A análise permitiu concluir que a maioria das recomendações estão em consonância com os preceitos para } \\
\text { a experiência positiva na perinatalidade, mas carecem de adaptação ao contexto brasileiro. }\end{array}$ \\
\hline
\end{tabular}

Fonte: Santana GCDS, et al., 2021. 
Observou-se que o comparecimento às consultas diminuiu durante a pandemia, assim todo um plano de consulta especializado foi criado e estabelecido por diversas instituições para evitar o contágio e a disseminação da doença, principalmente em mulheres grávidas que são grupo de risco (OZALP M, et al., 2020). Logo, tendo em vista a importância da continuidade do pré-natal mesmo durante a pandemia do COVID-19, para evitar complicações mais graves, foi visto a real necessidade da criação de novas ferramentas para serem implementadas no atendimento à grávida (MADDEN N, et al., 2020).

Quanto aos fatores direcionados para essas estratégias utilizadas na assistência ao pré-natal, as publicações argumentam sobre a imprescindibilidade da qualificação de todos os profissionais da área da saúde para atender as gestantes com segurança e qualidade, assim os artigos trazem e recomendam a prática dos programas de telemedicina (consulta por vídeo, áudios, uso de aplicativos) para agregar juntamente com a consulta presencial que neste caso são programadas com um número reduzido de pacientes e com a recomendação de utilização da paramentação indicada, tanto para o profissional quanto para pacientes e acompanhantes (AZIZ A, et al., 2020; NAKAGAWA K, et al., 2020).

\section{A importância do pré-natal e suas mudanças}

Sabe-se que o pré-natal é o acompanhamento indicado para todas as gestantes e que deve ser realizado durante todas as fases da gestação, desde a concepção até o período pós-parto. Desta forma o objetivo do pré-natal é conhecer todo o histórico da gestante, identificando as doenças pré-existentes, para então possibilitar uma gestação mais segura, tanto para a gestante como para seu bebê, fazendo com que sejam efetuadas ações e intervenções preventivas (MINISTÉRIO DA SAÚDE, 2013).

Vale ressaltar que ao chegar em um consultório a gestante deve ser bem acolhida e se sentir confortável, esse primeiro momento será crucial para a criação do vínculo entre o profissional e a usuária. Posteriormente, vem a anamnese que é a etapa onde serão coletadas todas as informações cruciais de vida da gestante, identificação, dados socioeconômicos e culturais, antecedentes familiares, antecedentes ginecológicos, sexualidade, antecedentes obstétricos, e informações atuais a respeito da gestação, como sintomas, sensações, emoções e mudanças (SECRETÁRIA DA SAÚDE DO ESTADO DO CEARÁ, 2014).

Em seguida, será feito o exame físico detalhado e minucioso, pois este é um momento importante para averiguar o estado de saúde da mãe e bebê, verificando intercorrências e o estado da gravidez, assim como também exames mais específicos e complementares serão realizados para prevenir e detectar patologias prévias, desta forma garantindo uma gestação com desenvolvimento saudável (MINISTÉRIO DA SAÚDE, 2000).

No que diz respeito ao atendimento presencial, os profissionais devem seguir algumas regras, principalmente quanto a paramentação adequada da equipe que prestará o atendimento, máscaras obrigatórias para os profissionais, gestante e acompanhante, além do distanciamento social. Na primeira consulta, ideal entre a $11^{\mathrm{a}}$ e a $14^{\mathrm{a}}$ semana deve ser feita a anamnese, o exame físico geral e obstétrico, avaliando a idade gestacional, a Data Provável do Parto (DPP), solicitar exames de rotina do 1ํㅡㄹ trimestre e a ultrassonografia, já da $20^{\underline{a}}$ a $22^{\underline{a}}$ semana, será um momento para a avaliação da anatomia fetal através da ultrassonografia morfológica (MINISTÉRIO DA SAÚDE,2020).

\section{COVID-19 e as suas consequências}

Inesperadamente, em 2020 o mundo inteiro foi surpreendido pela COVID-19, a doença infecciosa surgiu em Wuhan na China no final de 2019, e em março do mesmo ano a World Health Organization (WHO) declarou estado de pandemia. Dentre os principais sintomas podem ser destacados, febre, cansaço, tosse seca, perda de paladar e/ou olfato, alguns pacientes evoluem para quadros mais graves com dispneia e possível comprometimento pulmonar. Medidas sanitárias tiveram que ser adotadas em todo o mundo para a proteção individual e coletiva, como exemplo o isolamento social, uso de máscaras e práticas de higiene das mãos (WHO, 2020).

As formas de transmissão da doença ocorrem principalmente por contato direto por meio de gotículas e aerossóis que são expelidos pela pessoa infectada por meio de espirro, tosse ou no momento da fala sem as 
máscaras de proteção (ESAKANDARI $\mathrm{H}$, et al., 2020). O contato indireto, apesar de ser mais raro também pode ocorrer, isto é, quando a pessoa entra em contato com uma superfície contaminada por gotículas respiratórias e em seguida toca boca, nariz ou olhos sem higienizar as mãos (WIERSINGA WJ, et al., 2020).

Com o crescimento do número de casos em todo o mundo, pesquisas observaram que houve um aumento de casos de complicações maternas principalmente no último trimestre da gestação, puerpério e em gestantes que apresentam alguma comorbidade pré gestacional como, doenças cardiovasculares, renais, diabetes mellitus, hipertensão arterial grave, pneumonias crônicas graves e em imunossuprimidas. Dentre as complicações pode-se destacar, pré-eclâmpsia, eclampsia, síndrome de HELLP, admissão na unidade de terapia intensiva e parto prematuro (VILLAR J, et al., 2021). Também foram relatados casos de morte materna por Síndrome Respiratória Aguda Grave (SRAG), onde observou-se que o maior número de óbitos foi decorrente da infecção pelo SARS-CoV-2, durante o ano de 2020 (MINISTÉRIO DA SAÚDE, 2020).

O aumento da morbimortalidade nas gestante durante a pandemia no Brasil já vem sendo relatado na literatura, estudos demonstraram que 8 a cada 10 mortes maternas por COVID-19 no mundo acontecem no Brasil, e um desses fatores se deve a falha na assistência às gestantes, como a baixa qualidade no pré-natal principalmente por dificuldade de acesso, esses problemas já fazem parte do cotidiano, porém com a doença muitos serviços vem adotando protocolos restritivos que acabam dificultando mais ainda o acesso dessas gestantes (SANDRO A, et al., 2021). Atrelado a tais aspectos, segundo a Organização Mundial da Saúde (OMS), durante a gestação a mulher deve ter acesso a cuidados específicos e de qualidade, os cuidados prénatais, que exercem o papel de cuidados de saúde, incluindo a promoção da saúde, o rastreio, o diagnóstico e a prevenção de doenças (OMS, 2016).

O Ministério da saúde considera as gestantes e puérperas até o $14^{\circ}$ dia de pós-parto como grupo de risco frente a COVID-19, e medidas foram adotadas visando a proteção dessas mulheres durante a pandemia, as principais medidas foram relacionadas aos profissionais de saúde que atendem a essa população, o Manual de Recomendações para a Assistência à Gestante e Puérpera do MS traz informações sobre transmissão, prevenção, paramentação e assistência durante esse período (MINISTÉRIO DA SAÚDE, 2020).

Outro importante ponto a ser abordado é quanto a vacinação para as gestantes, puérperas e lactentes. $O$ Ministério da Saúde recomenda a vacinação de gestantes e puérperas até 45 dias como descrito no Plano Nacional de Operacionalização da Vacinação contra a COVID-19, a vacina pode ser oferecida após a avaliação de riscos e benefícios nessas mulheres que fazem parte de um dos grupos prioritários dentro do Plano Nacional de Imunização (PNI).Os profissionais devem informar as mesmas sobre as limitações no estudos quanto à eficácia e segurança das vacinas para esse grupo, além de orientações sobre a não interrupção da amamentação (MINISTÉRIO DA SAÚDE, 2021).

\section{Estratégias utilizadas no pré-natal durante a pandemia do COVID-19}

A vulnerabilidade das gestantes na pandemia é uma realidade, o momento da gestação já acarreta muitas mudanças que podem comprometer a saúde mental dessas mulheres, neste sentido a pandemia vem como mais um elemento que pode causar ou aumentar o sofrimento psíquico. A ansiedade, o medo de serem infectadas, a falta de informações e ou acesso a essas informações, a hora do parto, a presença ou não de acompanhante, as dúvidas sobre amamentação e as mudanças gerais da assistência são problemas reais quem vem afetando a saúde dessas mulheres (HERMANN A, et al., 2020).

Dessa forma, muitos serviços vêm adotando a modalidade virtual para auxiliar nesse processo contínuo de informação e troca de experiências. Consultas do pré-natal, grupos de gestantes, e essas vivências compartilhadas têm ajudado muitas mulheres a passarem por esse momento com um sentimento de acolhimento, mesmo que de uma forma virtual (SOUZA JB, et al., 2020).

Segundo a Federação Brasileira das Associações de Ginecologia e Obstetrícia (FEBRASGO) a consulta de pré-natal deve ser seguida normalmente conforme a idade gestacional da gestante e de acordo com o seu perfil de risco. No atendimento é recomendado que o profissional verifique a presença de sintomas do COVID-19 e faça com que a consulta seja a mais dinâmica possível para que a permanência seja a mínima 
do tempo necessário para a realização do pré-natal, assim impedindo que as salas de espera estejam cheias. Recomenda-se também que sejam estendidos os intervalos entre consultas e exames para assim, então, evitar a exposição das grávidas em lugares onde pode ser alto o risco de contágio (FEBRASGO, 2020).

Para a maior segurança de todos que irão estar presentes na consulta de pré-natal recomenda-se o uso de máscara apropriada para a gestante, acompanhante e o profissional também deve de máscara, avental ou jaleco, além disso a viseira de proteção (MINISTÉRIO DA SAÚDE, 2020). Recomenda-se o uso de álcool em gel em todos os atendimentos na sala de espera, nos consultórios e nas salas de exame, tanto na entrada quanto na saída (FEBRASGO, 2020).

De acordo com a Fundação Oswaldo Cruz (FIOCRUZ), caso a gestante apresente sintomas gripais e ou teste positivo para o COVID-19 a recomendação é que a consulta de pré-natal seja adiada e ela continue em seu isolamento. A grávida precisa ser avaliada de forma intensa pela equipe que utilizará ferramentas alternativas para o seu cuidado, afinal a mulher grávida precisa de um intensivo monitoramento, por exemplo da sua frequência respiratória, sinais vitais, sintomas presentes, saturação, pressão arterial, entre outros (FIOCRUZ, 2020).

Em concordância ao que foi demonstrado, o autor refere que para integrar com o atendimento presencial a telemedicina está sendo uma alternativa muito utilizada em instituições pelo mundo, o atendimento de telessaúde como a teleconsulta, teleorientação e o telemonitoramento que visam dar continuidade ao prénatal reduzindo o risco de exposição para as gestantes (HOLCOMB D, et al., 2020). Com o uso de aplicativos de vídeo ou ligações por áudio as consultas presenciais podem ser reduzidas pela metade para a gravidez de baixo risco, principalmente durante o primeiro trimestre da gravidez (AZIZ A, et al., 2021).

Ainda segundo o mesmo autor, quando a gestação for menor que 11 semanas a primeira consulta será virtual, onde o profissional irá realizar toda a anamnese e passará informações à gestante, seguida por uma consulta presencial para realização de exames a partir da 14 semana. Com isso, são realizadas consultas subsequentes alternando-as entre encontros virtuais e presenciais. Para gestantes que apresentam algum fator de risco associado e em gestações de alto risco o cuidado prestado deve ser mais criterioso em relação às consultas virtuais e aos cuidados durante o comparecimento aos serviços de saúde ( $A Z I Z A$, et al., 2021).

Diante dos artigos foi evidenciado que os profissionais devem ser qualificados e em algumas situações são treinados para prestar essa assistência virtual, com o auxílio de cursos de como utilizar os sistemas, protocolos de atendimento são criados a fim de conseguir realizar um atendimento de forma não presencial. As gestantes são orientadas durante todo o processo, sobre como utilizar as plataformas de teleatendimento, recebem ligações, mensagens ou e-mails com o passo a passo para os atendimentos (SAT SO e SOZBIR SY, 2021).

Os estudos encontrados descrevem uma variedade de programas e aplicativos, utilizados para o teleatendimento assim como os protocolos de atendimento das diversas instituições, porém todos apresentam respostas similares, como a continuidade da assistência, incorporando consultas virtuais e presenciais, o suporte tanto para a saúde física como mental reduzindo principalmente a ansiedade quanto ao medo da exposição ao vírus e ao mesmo tempo a preocupação com o bebê e ao não comparecimento às consultas (SAT SO e SOZBIR SY, 2021).

\section{CONSIDERAÇÕES FINAIS}

Diante da análise dos estudos, observou-se que com o surgimento da pandemia do COVID-19, foi necessário a inserção de novas estratégias para complementar a assistência ao pré-natal. Com isso, notouse que a telemedicina, a reorganização das consultas presenciais, a paramentação e qualificação dos profissionais são as estratégias utilizadas, e que levam a resultados positivos, favorecendo assim a diminuição da propagação do vírus e o aumento da promoção da saúde das gestantes. Porém, são necessários mais estudos sobre a temática, visto que os mesmos irão contribuir na capacitação de profissionais e estudantes da área de saúde, auxiliando no processo de cuidado, para que seja oferecida uma assistência segura e respaldada na literatura científica. 


\section{REFERÊNCIAS}

1. ARAÚJO D, et al. Atenção à saúde da mulher no pré-natal e puerpério em tempos de COVID-19: Uma revisão descritiva. Research, Society and Development, 2020; 9(9): 1-17.

2. AZIZ A, et al. Telehealth for High-Risk Pregnancies in the Setting of the COVID-19 Pandemic. Am J Perinatol, 2020; 37(8): 800-808.

3. BARBOSA-LEIKER C, et al. Stressors, coping, and resources needed during the covid-19 pandemic in a sample of perinatal women. BMC Pregnancy and Childbirth, 2021;171: 1-13.

4. BOTELHO LLR, et al. O método da revisão integrativa nos estudos organizacionais. Gestão e Sociedade, 2011; 5(11): 121-136.

5. BRASIL. Resolução CNS no 466, de 12 de dezembro de 2012. Aprova diretrizes e normas regulamentadoras de pesquisas envolvendo seres humanos. Diário Oficial da União, Poder executivo, Brasília, DF, jun. 2013.

6. CHATWIN J, et al. Experiences of pregnant mothers using a social media based antenatal support service during the COVID-19 lockdown in the UK: Findings from a user survey. BMJ Open, 2021;11(1): 1-7.

7. CHISINI LA, et al. Impact of the COVID-19 pandemic on prenatal, diabetes and medical appointments in the Brazilian National Health System. Rev. Bras. Epidemiol., 2021;24: 1-12.

8. DURYEA EL, et al. Comparison between in-person and audio-only virtual prenatal visits and perinatal outcomes. JAMA Netw Open, 2021;4(4): e215854.

9. ESAKANDARI H, et al. A comprehensive review of COVID-19 characteristics. Biol Proced Online, 2020; 22(19).

10. FEDERAÇÃO BRASILEIRA DAS ASSOCIAÇÕES DE GINECOLOGIA E OBSTETRÍCIA (FEBRASGO). Recomendações FEBRASGO para o GO em tempos de COVID-19. 2020. Disponível em: https://www.febrasgo.org.br/es/COVID19/item/975-recomendacoes-febrasgo-para-o-go-em-tempos-de-COVID-19. Acesso em: 24 jun. 2020.

11. FUNDAÇÃO OSWALDO CRUZ (FIOCRUZ). Principais questões sobre COVID-19 e gestação: atenção pré-natal e em maternidades. 2020. Disponível em: https://portaldeboaspraticas.iff.fiocruz.br/atencao-mulher/principais-questoesCOVID-19-gestacao-atencao-pre-natal-e-em-maternidades/. Acesso em: 20 ago. 2021.

12. GUTIÉRREZ MM, et al. A new multiplatform model for outpatient prenatal and postpartum care in a cohort of covid-19affected obstetric patients. Int. J. Environ. Res. Public Health, 2021; 18(10):1-13.

13. HERMANN A, et al. Meeting maternal mental health needs during the COVID-19 Pandemic. JAMA Psychiatry, 2021; 78(2): 123-124.

14. HOLCOMB D, et al. Patient perspectives on audio-only virtual prenatal visits amidst the severe acute respiratory syndrome coronavirus 2 (SARS-CoV-2) pandemic. Obstetrics and Gynecology, 2020;136(2): 317-322.

15. JEGANATHAN S, et al. Adherence and acceptability of telehealth appointments. American journal of obstetrics \& gynecology MFM, 2020;2: 1-9.

16. LEE T, et al. The outbreak of coronavírus disease in China: risk perceptions, knowledge, and information sources among prenatal and postnatal women. Women Birth, 2021;34(3): 212-218.

17. LÉLIS BDB, et al. O sofrimento mental das gestantes em meio a pandemia do novo coronavírus no Brasil. Revista de Psicologia, 2020; 14(52): 442-451.

18. MADDEN N, et al. Telehealth uptake into prenatal care and provider attitudes during the covid-19 pandemic in new city: quantitative and qualitative analysis. Am J perinatol, 2020;37(10): 1005-1012.

19. MINISTÉRIO DA SAÚDE. Cadernos de Atenção Básica, n 32: Atenção ao pré-natal de baixo risco. 2012. Disponível em: https://bvsms.saude.gov.br/bvs/publicacoes/cadernos_atencao_basica_32_prenatal.pdf. Acesso em: 15 ago. 2020.

20. MINISTÉRIO DA SAÚDE. Secretária de Atenção à Saúde. Gravidez, parto e nascimento com saúde, qualidade de $\begin{array}{llll}\text { vida } & \text { e } & 2013 . & \text { Dem-estar. }\end{array}$ http://bvsms.saude.gov.br/bvs/publicacoes/gravidez_parto_nascimento_saude_qualidade.pdf. Acesso em: 15 ago. 2020.

21. MINISTÉRIO DA SAÚDE. Secretária de Atenção Primária à Saúde. Manual de Recomendações para a Assistência à Gestante e Puérpera frente à Pandemia de COVID-19. 2020. Disponível em: https://portaldeboaspraticas.iff.fiocruz.br/atencao-mulher/manual-de-recomendacoes-para-a-assistencia-a-gestantee-puerpera-frente-a-pandemia-de-COVID-19/. Acesso em: 10 set. 2020.

22. MINISTÉRIO DA SAÚDE. Secretária de Políticas de Saúde. Assistência Pré-natal: Manual técnico. 2000. Disponível em: https://bvsms.saude.gov.br/bvs/publicacoes/cd04_11.pdf. Acesso em: 10 set. 2020.

23. MINISTÉRIO DA SAÚDE. Secretária extraordinária de enfrentamento à COVID-19. 2021. Disponível em: https://sbim.org.br/images/files/notas-tecnicas/sei-ms--0021464579--nota--tecnica-gestantes.pdf. Acesso em: 20 ago. 2021.

24. NAKAGAWA K, et al. Feasibility and safety of urgently initiated maternal telemedicine in response to the spread of COVID-19: A 1-month report. J Obstet Gynaecol Res, 2020;46(10): 1967-1971. 
25. ORGANIZAÇÃO MUNDIAL DA SAÚDE (OMS). Recomendações da OMS sobre cuidados pré-natais para uma experiência positiva na gravidez. 2016. Disponível em: https://apps.who.int/iris/bitstream/handle/10665/250800/WHORHR-16.12-por.pdf?sequence=2. Acesso em: 15 mar. 2021.

26. OZALP M, et al. Effect of COVID-19 pandemic process on prenatal diagnostic procedures. J Matern Fetal Neonatal Med, 2020;1: 1-6.

27. SANDRO A, et al. Mortalidade materna pela COVID-19 no Brasil. Rev. Bras. Saúde Matern. Infant, 2021; 21(1): 257261.

28. SAT SO, SÖZBIR ŞY. Use of mobile applications by pregnant women and levels of pregnancy distress during the COVID-19 (Coronavirus) Pandemic. Maternal and Child Health Journal, 2021;25(7): 1057-1068.

29. SECRETÁRIA DA SAÚDE DO ESTADO DO CEARÁ. Protocolos de obstetrícia da secretária da saúde do estado do Ceará. 2014. Disponível em: https://www.researchgate.net/profile/Deivide-SousaOliveira/publication/334596562_protocolos_obstetricia_sesa_ce_2014/links/5d34101292851cd04678b2dc/protocolos -obstetricia-sesa-ce-2014.pdf\#page=18. Acesso em: 28 abr. 2021.

30. SMITH RB, et al. Mindfulness effects in obstetric and gynecology patients during the coronavirus disease 2019 (COVID19) pandemic: a randomized controlled trial. Obstetrics \& Gynecology, 2021; 137(6):1032-1040.

31. SOUZA JB, et al. Reflexões sobre o enfrentamento do coronavírus disease 2019: diálogos virtuais com gestantes. Revista de Enfermagem do Centro-Oeste Mineiro, 2020; 10: e3792.

32. SOUZA MT, et al. Revisão integrativa: o que é e como fazer. Einstein, 2010; 8(1): 102-106.

33. STOFEL NS, et al. Perinatal care in the COVID-19 pandemic: Analysis of Brazilian guidelines and protocols. Revista Brasileira de Saude Materno Infantil,2021;21(1): 89-98.

34. TOMAZETTI BM, et al. A qualidade da assistência pré-natal sob olhar multiprofissional. A Ciência \& Saúde, 2018; 11(1): 41-50.

35. VILLAR J, et al. Maternal and Neonatal Morbidity and Mortality Among Pregnant Women With and Without COVID-19 Infection: The INTERCOVID Multinational Cohort Study. JAMA pediatrics, 2021; 175(8): 817-826.

36. WIERSINGA WJ, et al. Pathophysiology, Transmission, Diagnosis, and Treatment of Coronavirus Disease 2019 (COVID-19): A Review. Journal of the American Medical Association, 2020; 324(8): 782-793.

37. WORLD HEALTH ORGANIZATION (WHO). Coronavirus disease (COVID-19). 2020. Disponível em: https://www.who.int/emergencies/diseases/novel-coronavirus-2019/question-and-answers-hub/q-a-detail/coronavirusdisease-COVID-19. Acesso em: 10 dez. 2020. 